\title{
Comments on "A Semiotic Approach to the Design of Agent-mediated E-commerce Systems" by S. Chong and K. Liu
}

\section{John Krogstie}

Andersen Consulting and IDI, Norwegian University of Science and Technology (NTNU), Norway

In the nineties, several new technologies and technological possibilities have been established, such as ERP-systems (e.g. SAP and Baan) and internet-technology spawning increasing possibilities in areas such as eCommerce. In general, the academic world is rather slow in addressing the problems and opportunities of new commercial concepts and technologies, although the general frameworks and ideas developed in the scientific community often have a lot to offer for understanding the development and use of new technologies in practice. The paper by Chong and Liu is an attempt on such cross-fertilisation, using the academically devised organisational semiotics in connection to eCommerce-systems, systems that are of increasing importance in most modern organisations.

ECommerce can be defined as any interaction over an electronic network between two or more computers or electronic interfaces, where:

- Information/media, services or physical goods are traded for financial or other consideration.

- Payment is accomplished electronically either through the same or a parallel network

The paper addresses (requirements) analysis (not design) of eCommerce systems, with particular emphasis on the effective use of agent-technology. Agents are one of many useful (but not mandatory) technologies that are applied as part of an eCommerce-solution. The "agent" label is stuck on many different kinds of software processes or systems to the point that it is now difficult to give a precise definition of what an agent is. 
A common idea is that an agent is software that acts on a user's behalf and achieves tasks a user could achieve if he had enough time. Although delegation is mentioned in the paper, it is not included as part of the definition of the term, giving a definition of agent that can be used for almost anything. It could be useful for the further work of the authors for them to define which agent characteristics and agent types they are supporting.

I use ideas of organisational semiotics and the semiotic ladder in many circumstances myself, but have some comments on the way it is used in the paper. When evaluating KQML only the three upper levels (semantics, pragmatics, and social) are addressed. I understand that this is a conscious decision by the authors, but think that this a sub-optimal way of using the semiotic ladder.

The critique of KQML at the semantic level (and indirectly pragmatic level), can be summarised with: 'KQML, like all programming languages do not have a formal and precise semantic meaning of the performatives..... As a result agent designers do not have a common understanding of the language, its performatives and protocol." A KQML-message is usually one piece of dialogue, consisting of a communications type and one or more KIF expressions. KIF is an enhanced version of the language of first-order predicate calculus, which does have a formal (mathematical) semantics, which is quite well known and used in practice. Obviously, using a language with mathematical foundations only addresses the semantical issues when dealing with simple problems where the people involved share a wellestablished consensus about the task at hand. This is not the case when taking about eCommerce systems, were systems that eventually will need to communicate can be developed anywhere in the world. On the other hand, it is very difficult to see how the work described in the paper can help in this regard, since it present a language for which not even a formal syntax is developed for important parts of the language.

More interesting then is the discussion on language appropriateness linked up to the social level, i.e. to what extent it is possible to express what one would like to express in connection to the development of agents. This is used partly on the basis of an extended list of communicative acts (the traditional five from Searle extended with two deontic notions, 'permissives' and 'prohibitives'), and partly using the ideas of speech acts in the similar way as they are used in Action Workflow. Although pointing to some weaknesses in KQML, it is not spelled out in detail how the proposed 'semiotic' methods address these and other needs. It is generally difficult to see to what extent the proposed language is adapted to agent and eCommerce-systems. Another open point is how to sensibly formalise the proposed framework, which include fuzzy concepts such as 'attitudes' and 'dispositions'. 